\title{
ON FIXED POINT THEORY AND ITS APPLICATIONS TO EQUILIBRIUM MODELS
}

D.A. Serkov, Krasovskii Institute of Mathematics and Mechanics, Ural Federal University named after the first President of Russia B.N. Yeltsin, Yekaterinburg, Russian Federation, serkov@imm.uran.ru

For a given set and a given (generally speaking, multivalued) mapping of this set into itself, we study the problem on the existence of fixed points of this mapping, i.e., of points contained in their images. We assume that the given set is nonempty and the given mapping is defined on the entire set. In these conditions, we give the description (redefinition) of the set of fixed points in the set-theoretic terms. This general idea is concretized for cases where the set is endowed with a topological structure and the mapping has additional properties associated with this structure. In particular, we provide necessary and sufficient conditions for the existence of fixed points of mappings with closed graph in Hausdorff topological spaces as well as in metric spaces. An example illustrating the possibilities and advantages of the proposed approach is given. The immediate applications of these results to the search of equilibrium states in game problems are also given: we describe the sets of saddle points in the minimax problem (an analogue of the Fan theorem) and of Nash equilibrium points in the game with many participants in cases where the sets of strategies of players are Hausdorff spaces or metrizable topological spaces.

Keywords: multivalued mapping; fixed point; saddle point; Nash equilibrium.

\section{Introduction}

If one consider an automorphism $F$ of a set $X$ as the dynamics of a system with discrete time and the state space $X$, then many sufficient conditions for the existence of fixed points can be treated as conditions that prevent the formation of cycles (consisting of several points):

- "the contraction principle": the distance between the images of two arbitrary points is less than the distance between these points (a cycle has to be contracted to a point);

- "the directionality principle": the set $X$ is partially ordered and the mapping $F$ is isotonic with respect to this order (only single-point cycles are possible).

In this case, either the linear structure (see the Banach principle of contractive mappings, the Kakutani theorem [1], and their generalization [2]), or the order structure (see theorems by Tarski [3], Kantorovich [4], Kleene [5, Theorem 1.2.17], and their generalization [6]) on the set $X$ is used.

At the same time, in view of the known connection between fixed points and equilibrium points (see Lemmas 3 and 4), one would expect a fixed point result under conditions similar to the conditions of the Fan theorem [7]. We recall that this theorem is based only on topological properties of the domain of the quality function.

From this point of view, the idea of the conditions proposed in the paper consists in restricting the sizes of cycles due to the representation of the initial mapping by the set of its restrictions to a covering of its domain: a single-point cycle is present for any choice of such a covering; all other cycles, in dependence on the properties of the mapping (we require the closedness of the graph), are "cut off" under an appropriate choice of a family of coverings. 


\section{Definitions}

1. For an arbitrary set $X$ denote by $2^{X}$ the set of all subsets of $X$. Consider a nonempty set $X$ and a multivalued mapping $X \ni x \mapsto F(x) \in 2^{X}$. Denote by $\mathbf{F i x}(F)$ the set of all fixed points of the mapping $F: \operatorname{Fix}(F):=\{x \in X \mid x \in F(x)\}$. For the given mapping $F$, define the mapping $2^{X} \ni Y \mapsto \hat{F}(Y) \in 2^{X}$ as follows:

$$
\hat{F}(Y):=\left(\bigcup_{y \in Y} F(y)\right) \bigcap Y=\bigcup_{y \in Y} F(y) \cap Y .
$$

Observe that $\hat{F}(Y)$ is the set of "candidates" from a set $\mathrm{Y}$ for the inclusion in the set of fixed points: by contradiction, it is easy to verify that the elements of $Y$ not lying in $\hat{F}(Y)$ obviously do not belong to $\mathbf{F i x}(F)$. In particular, for any singleton set $\{x\} \in 2^{X}, x \in X$, the set $\hat{F}(\{x\})$ is nonempty if and only if $x \in \mathbf{F i x}(F)$.

Immediately from the definition, for any $Y, Y^{\prime} \in 2^{X}$, we obtain

$$
\begin{gathered}
\hat{F}(Y) \subset Y, \\
Y^{\prime} \subset Y \Rightarrow \hat{F}\left(Y^{\prime}\right) \subset \hat{F}(Y), \\
\mathbf{F i x}(F) \cap Y=\mathbf{F i x}(F) \cap \hat{F}(Y), \\
(Y \subseteq \operatorname{Fix}(F)) \Rightarrow(Y \in \mathbf{F i x}(\hat{F})) .
\end{gathered}
$$

From the definition of $\hat{F}$ and the above relations, for an arbitrary family $\left(Z_{\tau}\right)_{T} \subset 2^{X}$, we get

$$
\begin{gathered}
\bigcup_{\tau \in T} \hat{F}\left(Z_{\tau}\right) \subset \bigcup_{\tau \in T} Z_{\tau}, \\
\operatorname{Fix}(F) \bigcap \bigcup_{\tau \in T} Z_{\tau}=\mathbf{F i x}(F) \bigcap \bigcup_{\tau \in T} \hat{F}\left(Z_{\tau}\right) .
\end{gathered}
$$
(3).

Here, inclusion (4) follows from inclusion (1) and equality (5) follows from equality

2. For families in $2^{X}$ define the relation to be inscribed which will be denoted by the symbol $\sqsubseteq$ : for arbitrary $\left(Z_{\tau}\right)_{T},\left(Z_{\tau^{\prime}}^{\prime}\right)_{T^{\prime}} \subset 2^{X}$, we say that the family $\left(Z_{\tau^{\prime}}^{\prime}\right)_{T^{\prime}}$ is inscribed into the family $\left(Z_{\tau}\right)_{T}$ and denote this as $\left(Z_{\tau^{\prime}}^{\prime}\right)_{T^{\prime}} \sqsubseteq\left(Z_{\tau}\right)_{T}$ if, for arbitrary $\tau^{\prime} \in T^{\prime}$, there exists $\tau \in T$ such that $Z_{\tau^{\prime}}^{\prime} \subset Z_{\tau}$. In the sequel, this order relation will be useful in view of the following property of the mapping $\hat{F}$ : it follows from implication (2) that if $\left(Z_{\tau^{\prime}}^{\prime}\right)_{T^{\prime}},\left(Z_{\tau}\right)_{T} \subset 2^{X}$ and $\left(Z_{\tau^{\prime}}^{\prime}\right)_{T^{\prime}} \sqsubseteq\left(Z_{\tau}\right)_{T}$, then

$$
\bigcup_{\tau^{\prime} \in T^{\prime}} \hat{F}\left(Z_{\tau^{\prime}}^{\prime}\right) \subset \bigcup_{\tau \in T} \hat{F}\left(Z_{\tau}\right)
$$

3. Denote by $O(X)$ the set of all coverings of $X$, i.e., the set of all families $\left(O_{\iota}\right)_{I} \subset 2^{X}$ such that $\cup_{\iota \in I} O_{\iota}=X$. Let $\tau(X)$ be a topology in $X$ (the family of all open sets). Denote by $O_{\mathrm{fo}}(X)\left(O_{\mathrm{fc}}(X)\right)$ the set of all finite open (closed) coverings of $X$. 


\section{Criterion for the Existence of Fixed Points}

1. The set of fixed points of the mapping $F$ can formally be described as follows.

Theorem 1. For any nonempty set $X$ and for any mapping $F: X \mapsto 2^{X}$, we have

$$
\boldsymbol{F i x}(F)=\bigcap_{\left(O_{\iota}\right)_{I} \in O(X)} \bigcup_{\iota \in I} \hat{F}\left(O_{\iota}\right) .
$$

Remark 1. Since among the coverings there exists a smallest element with respect to the inscribing relation, the covering $(\{x\})_{X}$ consisting of all singleton sets of the set $X$, by (6), for this covering and for any $\left(O_{\iota}\right)_{I} \in O(X)$, we have $\bigcup_{x \in X} \hat{F}(\{x\}) \subset \bigcup_{\iota \in I} \hat{F}\left(O_{\iota}\right)$ and, hence, $\operatorname{Fix}(F)=\bigcup_{x \in X} \hat{F}(\{x\})$. However, exactly relation (7) is important for us, since it gives possibilities for the use of properties of the set $X$ and the mapping $F$.

2. The corollaries of equality (7) given below are based on the intuitively obvious fact that if a closed subset $G$ of a compact set $X \times X$ has points arbitrarily close to the diagonal $\{(x, x) \mid x \in X\}$, then $G$ contains an element $(\bar{x}, \bar{x})$ of the diagonal. At the same time, if the set $G$ is the graph of a multivalued mapping $F$, then the element $\bar{x}$ is a fixed point of the mapping $F$.

Theorem 2. Let $X$ be a compact Hausdorff space, and let a mapping $F$ have the closed graph. Then

$$
\boldsymbol{F i x}(F)=\bigcap_{\left(O_{\iota}\right)_{I} \in O_{\mathrm{fo}}(X)} \bigcup_{\iota \in I} \hat{F}\left(O_{\iota}\right)=\bigcap_{\left(O_{\iota}\right)_{I} \in O_{\mathrm{fc}}(X)} \bigcup_{\iota \in I} \hat{F}\left(O_{\iota}\right) .
$$

In particular, the set $\mathbf{F i x}(F)$ is nonempty if and only if the following condition holds:

$$
\left(\forall\left(O_{\iota}\right)_{I} \in O_{\mathrm{fc}}(X)\right)(\exists \bar{\iota} \in I) \quad \hat{F}\left(O_{\bar{\iota}}\right) \neq \varnothing .
$$

Corollary 1. Let $X$ be a compact Hausdorff space, and let a function $f: X \mapsto X$ be continuous. Then $\boldsymbol{F i x}(f)=\bigcap_{\left(O_{\iota}\right)_{I} \in O_{\mathrm{fo}}(X)} \bigcup_{\iota \in I} \hat{f}\left(O_{\iota}\right)=\bigcap_{\left(O_{\iota}\right)_{I} \in O_{\mathrm{fc}}(X)} \bigcup_{\iota \in I} \hat{f}\left(O_{\iota}\right)$. In particular, the function $f$ has a fixed point if and only if $\left(\forall\left(O_{\iota}\right)_{I} \in O_{\mathrm{fc}}(X)\right)(\exists \bar{\imath} \in I)$ $\hat{f}\left(O_{\bar{\iota}}\right) \neq \varnothing$.

3. Let the space $X$ be endowed with a metric $\rho: X^{2} \mapsto[0, \infty)$. Denote by $\mathbf{d}(x, A)$ the distance from a point $x \in X$ to a set $A \subset X$ defined by the metric $\rho: \mathbf{d}(x, A):=\inf _{a \in A} \rho(a, x)$. For any $Y \in 2^{X}$, define the diameter of the set $Y$ : $\operatorname{diam}(Y):=\sup _{y, y^{\prime} \in Y} \rho\left(y, y^{\prime}\right)$. Assume that the topology $\tau(X)$ generated by the metric $\rho$ is defined in $X$. For any $\delta>0$, denote by $O_{\mathrm{fc}}^{\delta}(X)\left(O_{\mathrm{fo}}^{\delta}(X)\right)$ the subset of $O_{\mathrm{fc}}(X)\left(O_{\mathrm{fo}}(X)\right)$ of closed (open) finite coverings $\left(O_{\iota}\right)_{I}$ of diameter not greater than $\delta: \max _{\iota \in I} \operatorname{diam}\left(O_{\iota}\right) \leqslant \delta$. Recall that if $X$ is a compact metric space, then for any $\delta>0$, the sets $O_{\mathrm{fc}}^{\delta}(X)$ and $O_{\mathrm{fo}}^{\delta}(X)$ are nonempty.

Theorem 3. Let $X$ be a compact metric space, and let a mapping $F$ have the closed graph. Then, for an arbitrary family of coverings

$$
\left(O_{k \iota}\right)_{I_{k}} \in O_{\mathrm{fc}}^{\delta_{k}}(X) \cup O_{\mathrm{fo}}^{\delta_{k}}(X), \quad \delta_{k}>0, \quad k \in \mathbb{N}, \quad \lim _{k \rightarrow \infty} \delta_{k}=0,
$$

the equality $\mathbf{F i x}(F)=\bigcap_{k \in \mathbb{N}} \bigcup_{\iota \in I_{k}} \hat{F}\left(O_{k \iota}\right)$ holds. In particular, the set $\mathbf{F i x}(F)$ is nonempty if and only if

$$
(\forall \delta>0)\left(\exists x_{\delta} \in X\right) \quad \mathbf{d}\left(x_{\delta}, F\left(x_{\delta}\right)\right) \leqslant \delta .
$$


Corollary 2. Let $X$ be a compact metric space, and let a function $f$ be continuous. Then, for an arbitrary family of coverings of the form (10), the equality $\mathbf{F i x}(f)=$ $\bigcap_{k \in \mathbb{N}} \bigcup_{\iota \in I_{k}} \hat{f}\left(O_{k \iota}\right)$ holds. In particular, the function $f$ has a fixed point if and only if

$$
(\forall \delta>0)\left(\exists x_{\delta} \in X\right) \quad \rho\left(x_{\delta}, f\left(x_{\delta}\right)\right) \leqslant \delta .
$$

Remark 2. Theorems 2 and 3 as well as Corollaries 1 and 2 remain true if we replace the condition of compactness of the space $X$ by the condition of precompactness of the image of the space $X$ under the mapping $F(f)$ :

$$
\bigcup_{x \in X} F(x) \subset K \quad\left(\bigcup_{x \in X} f(x) \subset K\right),
$$

where $K \in 2^{X}$ is a compact set in the space $(X, \tau(X))$.

\subsection{Example}

We give as much as possible simple example of the use of Theorem 3. Consider the closed interval $[0,1]$ as a compact metric space $X$ with the natural metric $\rho(x, y):=|x-y|$. Choose and fix an infinite disjoint sequence $\left(\left[a_{i}, b_{i}\right]\right)_{\mathbb{N}}$ of closed intervals in $X$ of nonzero length: $a_{i}<b_{i},\left[a_{i}, b_{i}\right] \cap\left[a_{j}, b_{j}\right]=\varnothing, i, j \in \mathbb{N}, i \neq j$. Define the mapping $G: X \mapsto 2^{X}$, setting

$$
G(x):=\bigcup_{i \in \mathbb{N}} G_{\left[a_{i}, b_{i}\right]}(x), \quad G_{[a, b]}(x):= \begin{cases}\operatorname{argmax}_{y \in[a, b]}|y-x|, & x \in[a, b], \\ \varnothing, & x \notin[a, b] .\end{cases}
$$

Define the mapping $F: X \mapsto 2^{X}$ as follows: the graph of the mapping $F$ is the closure in $\mathbb{R}^{2}$ of the graph of the mapping $G$. Note that the values of mapping $F$ are nonconvex or empty for some elements $x \in X$.

It is easy to see that mapping $F$ satisfies conditions of Theorem 3: because of the disjointness of intervals $\left[a_{i}, b_{i}\right]$, their lengths tend to zero as $i \rightarrow \infty$ and, hence, for any $\delta>0$, the inequality $\mathbf{d}\left(a_{i}, F\left(a_{i}\right)\right) \leqslant \delta$ holds for a sufficiently large $i$. Consequently, by Theorem 3, the mapping $F$ has a fixed point. One can verify that all partial limits of the sequence $\left(a_{i}\right)_{\mathbb{N}}$ are fixed points. Certainly, such limits exist in view of the compactness of $X$.

In connection with the considered example, we also note that

- the mapping $F$ is not $k$-contractive (it suffices to consider the passage of the argument through the center of any interval $\left.\left[a_{i}, b_{i}\right]\right)$, and, therefore, the Nadler theorem [8] is not applicable;

- the mapping $F$ is not $\alpha$-covering, since is not surjective, and, hence, the theorem on coincidence points [9] is not applicable;

- the values of mapping $F$ are nonconvex and may take the value $\varnothing$; therefore, the Kakutani theorem [1] is not applicable.

\section{Applications to Equilibrium Theorems}

Using the known method connecting the equilibrium positions with fixed points of multivalued mappings (see Lemmas 3 and 4), we can obtain new versions of equilibrium 
theorems from the above theorems on fixed points. The very conditions describing the set of equilibriums are rather formal. However, their particular case, a property of Cournot approximations, may be of interest for applications.

1. From Theorem 2, we can obtain one more theorem on a criterion of the equality of the minimax and maximin values in conditions similar to the conditions of the Fan theorem [7].

Let $\mathbf{U}$ and $\mathbf{V}$ be (topological) spaces of strategies of two players, and let the standard topology of the product and an outcome function $\varphi: \mathbf{U} \times \mathbf{V} \mapsto \mathbb{R}$ with scalar values be given on the product $X:=\mathbf{U} \times \mathbf{V}$. The player choosing strategies $u \in \mathbf{U}$ tends to minimize the outcome of the game, and the player choosing strategies $v \in \mathbf{V}$ tends to increase the outcome. Define $S(\varphi) \in 2^{\mathbf{U}} \times 2^{\mathbf{V}}$ as the set of saddle points of function $\varphi$, i.e., as the set of pairs $\left(u_{*}, v_{*}\right) \in \mathbf{U} \times \mathbf{V}$ satisfying the conditions $\varphi\left(u_{*}, v\right) \leqslant \varphi\left(u, v_{*}\right) \forall(u, v) \in \mathbf{U} \times \mathbf{V}$. Consider the multivalued mapping $X \ni(u, v) \mapsto F_{\varphi}(u, v) \in 2^{X}$ of the form

$$
F_{\varphi}(u, v):=\underset{u^{\prime} \in \mathbf{U}}{\operatorname{argmin}} \varphi\left(u^{\prime}, v\right) \times \underset{v^{\prime} \in \mathbf{V}}{\operatorname{argmax}} \varphi\left(u, v^{\prime}\right), \quad(u, v) \in X .
$$

Theorem 4. Let $\mathbf{U}$ and $\mathbf{V}$ be compact Hausdorff spaces. Let, for any $v \in \mathbf{V}$, the function $\varphi(\cdot, v): \mathbf{U} \mapsto \mathbb{R}$ be lower semicontinuous on $\mathbf{U}$, and let, for any $u \in \mathbf{U}$, the function $\varphi(u, \cdot): \mathbf{V} \mapsto \mathbb{R}$ be upper semicontinuous on $\mathbf{V}$. Then, for the set $S(\varphi)$ of saddle points of the function $\varphi$, the following equalities hold:

$$
S(\varphi)=\operatorname{Fix}\left(F_{\varphi}\right)=\bigcap_{\left(O_{\iota}\right)_{I} \in O_{\mathrm{fo}}(X)} \bigcup_{\iota \in I} \hat{F}_{\varphi}\left(O_{\iota}\right)=\bigcap_{\left(O_{\iota}\right)_{I} \in O_{\mathrm{fc}}(X)} \bigcup_{\iota \in I} \hat{F}_{\varphi}\left(O_{\iota}\right) .
$$

In particular, the equality

$$
\max _{v \in \mathbf{V}} \min _{u \in \mathbf{U}} \varphi(u, v)=\min _{u \in \mathbf{U}} \max _{v \in \mathbf{V}} \varphi(u, v)
$$

holds if and only if for an arbitrary covering $\left(O_{\iota}\right)_{I} \in O_{\mathrm{fc}}(X)$, there exists a set $O_{\bar{\iota}}, \bar{\iota} \in I$, containing two successive Cournot approximations $x, x^{\prime} \in O_{\bar{\iota}}, x^{\prime} \in F_{\varphi}(x)$.

2. Passing to a more general case of the game, we obtain one more version of the theorem on the existence of Nash equilibrium. Let $(X, J)$ be a game with $n$ players in the normal form: $X:=X_{1} \times \ldots \times X_{n}$ and $J:=\left(J_{1}, \ldots J_{n}\right)$. Here, $J_{i}$ is the payoff function of the $i$ th player: $J_{i}: X \mapsto \mathbb{R}, i \in 1 . . n$, and in the case where $X_{i}$ are topological spaces, we assume that the standard topology of the product acts on $X$. Define $\left(y, x_{-i}\right):=\left(x_{1}, \ldots, x_{i-1}, y, x_{i+1}, \ldots, x_{n}\right), y \in X_{i}, x=\left(x_{1}, \ldots, x_{n}\right) \in X$. Denote by $N(J)$ the set of elements $x^{*} \in X$ for which the Nash equilibrium is attained, i.e., for which the following conditions are satisfied:

$$
J_{i}\left(y_{i}, x_{-i}^{*}\right) \leqslant J_{i}\left(x^{*}\right), \quad \forall y_{i} \in X_{i}, \forall i \in 1 . . n .
$$

By analogy with (13), introduce the multivalued mapping $X \ni x \mapsto F_{(X, J)}(x) \in 2^{X}$ :

$$
F_{(X, J)}(x):=\underset{y_{1} \in X_{1}}{\operatorname{argmax}} J_{1}\left(y_{1}, x_{-1}\right) \times \ldots \times \underset{y_{n} \in X_{n}}{\operatorname{argmax}} J_{n}\left(y_{n}, x_{-n}\right), \quad x \in X .
$$

Theorem 5. Let $X_{i}, i \in 1$..n, be compact Hausdorff spaces, and let, for all $x \in X$ and $i \in 1$..n, the function $J_{i}\left(\cdot, x_{-i}\right): X_{i} \mapsto \mathbb{R}$ be upper semicontinuous on $X_{i}$. Then, for the 
set $N(J)$ of Nash equilibriums, we have

$$
N(J)=\mathbf{F i x}\left(F_{(X, J)}\right)=\bigcap_{\left(O_{\iota}\right)_{I} \in O_{\mathrm{fo}}(X)} \bigcup_{\iota \in I} \hat{F}_{(X, J)}\left(O_{\iota}\right)=\bigcap_{\left(O_{\iota}\right)_{I} \in O_{\mathrm{fc}}(X)} \bigcup_{\iota \in I} \hat{F}_{(X, J)}\left(O_{\iota}\right) .
$$

In particular, Nash equilibrium (15) is attained if and only if for an arbitrary covering $\left(O_{\iota}\right)_{I} \in O_{\mathrm{fc}}(X)$, there exists a set $O_{\bar{\iota}} \in\left(O_{\iota}\right)_{I}$ containing two successive Cournot approximations:

$$
\left(\forall\left(O_{\iota}\right)_{I} \in O_{\mathrm{fc}}(X)\right)(\exists \bar{\iota} \in I)\left(\exists x, x^{\prime} \in O_{\bar{\iota}}\right) \quad x^{\prime} \in F_{(X, J)}(x) .
$$

Remark 3. In the case where the topology of the space $X$ is metrizable, condition (16) is naturally transformed into condition (11), taking the form $(\forall \delta>0)\left(\exists x_{\delta} \in X\right)$ $\mathbf{d}\left(x_{\delta}, F_{(X, J)}\left(x_{\delta}\right)\right) \leqslant \delta$.

Remark 4. Note that Theorem 5, as well as Theorem 4 and the Fan theorem [7], has the character of a criterion.

\section{Proofs}

\subsection{Proof of Theorem 1}

The inclusion $\operatorname{Fix}(F) \subset \bigcap_{\left(O_{\iota}\right)_{I} \in O(X)} \bigcup_{\iota \in I} \hat{F}\left(O_{\iota}\right)$ follows from (5). Indeed, by (5), for any $\left(O_{\iota}\right)_{I} \in O(X)$, we have the equalities $\boldsymbol{F i x}(F)=\boldsymbol{F i x}(F) \cap \bigcup_{\iota \in I} O_{\iota}=\mathbf{F i x}(F) \cap$ $\bigcup_{\iota \in I} \hat{F}\left(O_{\iota}\right)$ and, consequently, $\mathbf{F i x}(F) \subset \bigcup_{\iota \in I} \hat{F}\left(O_{\iota}\right)$.

Let us show the converse inclusion. Since $\{\boldsymbol{F i x}(F),\{x\} \mid x \in X, x \notin \mathbf{F i x}(F)\} \in O(X)$, the belonging of $\bar{x}$ to the right-hand side of equality (7) yields, in particular, the inclusion $\bar{x} \in \hat{F}(\mathbf{F i x}(F)) \cup \bigcup_{x \in X \backslash \mathbf{F i x}(F)} \hat{F}(\{x\})$ which, in view of $(1)$ and the equalities $\hat{F}(\{x\})=\varnothing$ for $x \in X \backslash \mathbf{F i x}(F)$, implies the inclusions $\bar{x} \in \hat{F}(\mathbf{F i x}(F)) \subset \mathbf{F i x}(F)$.

\subsection{Proof of Theorem 2 and Corollary 1}

By virtue of Theorem 1 and the inclusions $O_{\mathrm{fo}}(X), O_{\mathrm{fc}}(X) \subset O(X)$, for the proof of the assertion, it suffices to establish that

$$
\begin{aligned}
& \boldsymbol{F i x}(F) \supset \bigcap_{\left(O_{\iota}\right)_{I} \in O_{\mathrm{fo}}(X)} \bigcup_{\iota \in I} \hat{F}\left(O_{\iota}\right), \\
& \operatorname{Fix}(F) \supset \bigcap_{\left(O_{\iota}\right)_{I} \in O_{\mathrm{fc}}(X)} \bigcup_{\iota \in I} \hat{F}\left(O_{\iota}\right) .
\end{aligned}
$$

1. Justify inclusion (17). Let $\bar{x}$ belong to the right-hand side of (17). For an arbitrary local base of the topology $\left(O_{s}(\bar{x})\right)_{S}$ of the compact set $X$ at the point $\bar{x}$, we construct a family of coverings $\left(O_{s \iota}\right)_{I_{s}}, s \in S$, from $O_{\text {fo }}(X)$ such that, for any $s \in S$, we have

$$
\left(\exists \iota_{s} \in I_{s}: O_{s}(\bar{x})=O_{s \iota_{s}}\right) \&\left(\forall \iota \in I_{s} \backslash\left\{\iota_{s}\right\} \bar{x} \notin O_{s \iota}\right) .
$$

Fix an arbitrary $s \in S$ and, using the Hausdorff property, for every element $y \in X \backslash O_{s}(\bar{x})$, choose disjoint open neighborhoods $O_{s y}$ and $O_{s \bar{x}}$ of the elements $y$ and $\bar{x}$. The family

$$
\left\{O_{s}(\bar{x}), O_{s y} \mid y \in X \backslash O_{s}(\bar{x})\right\}
$$


is an open covering of $X$. For the chosen $s$, we take a finite subcovering of covering (20) as the covering $\left(O_{s \iota}\right)_{I_{s}}$. By construction, this covering has all the above properties.

Relations (4) and (19) along with the assumption that $\bar{x}$ belongs to the right-hand side of (8) imply that the inclusions

$$
\begin{aligned}
& \bar{x} \in \bigcup_{\iota \in I_{s}} \hat{F}\left(O_{s \iota}\right) \backslash \bigcup_{\substack{\iota \in I_{s} \\
\iota \neq \iota_{s}}} O_{s \iota} \subset \bigcup_{\iota \in I_{s}} \hat{F}\left(O_{s \iota}\right) \backslash \bigcup_{\substack{\iota \in I_{s} \\
\iota \neq \iota_{s}}} \hat{F}\left(O_{s \iota}\right) \subset \hat{F}\left(O_{s}(\bar{x})\right)= \\
& =\left(\bigcup_{y \in O_{s}(\bar{x})} F(y)\right) \bigcap O_{s}(\bar{x})
\end{aligned}
$$

hold for all $s \in S$.

Let us show that $\bar{x} \in \mathbf{F i x}(F)$. Assume the contrary: $\bar{x} \notin F(\bar{x})$. The closedness of the graph of $F$ yields the closedness of the set $F(\bar{x}) \subset X$. This, in view of the fact that $\mathrm{X}$ is a compact Hausdorff space, implies the existence of open neighborhoods $O^{\prime}(\bar{x})$ and $O^{\prime}(F(\bar{x}))$ of the point $\bar{x}$ and of the closed set $F(\bar{x})$ such that

$$
O^{\prime}(\bar{x}) \bigcap O^{\prime}(F(\bar{x}))=\varnothing .
$$

Since the graph of mapping $F$ is closed and $X$ is a compact Hausdorff space, the mapping $F$ is upper semicontinuous (see [10, Sect. 43, I, Theorem 4] and [11, Theorem 1.2.32]), i.e., for the neighborhood $O^{\prime}(F(\bar{x}))$, there exists a neighborhood $O^{\prime \prime}(\bar{x})$ such that, for all $y \in O^{\prime \prime}(\bar{x})$, we have

$$
F(y) \subset O^{\prime}(F(\bar{x})) \text {. }
$$

It follows from the definition of the family $\left(O_{s}(\bar{x})\right)_{s \in S}$ that the inclusion

$$
O_{\bar{s}}(\bar{x}) \subset O^{\prime}(\bar{x}) \cap O^{\prime \prime}(\bar{x})
$$

holds for some $\bar{s} \in S$. Relations (22), (23), and (24) yield the inclusions

$$
\left(\bigcup_{y \in O_{\bar{s}}(\bar{x})} F(y)\right) \bigcap O_{\bar{s}}(\bar{x}) \subset\left(\bigcup_{y \in O^{\prime \prime}(\bar{x})} F(y)\right) \bigcap O^{\prime}(\bar{x}) \subset O^{\prime}(F(\bar{x})) \bigcap O^{\prime}(\bar{x})=\varnothing
$$

which lead to the contradiction with (21) for $s=\bar{s}$.

2. To justify inclusion (18), we prove two auxiliary assertions.

Lemma 1. Let $X$ be a compact Hausdorff space. Then, for any element $x \in X$ and for any neighborhood $O \in \tau(X), x \in O$, there exist a closed set $B$ and a neighborhood $O^{\prime} \in \tau(X)$ such that $x \in O^{\prime} \subset B \subset O$.

Proof. Let $x \in O \in \tau(X)$. Since the set $X \backslash O$ is closed and does not contain $x$, by virtue of the fact that $X$ is a compact Hausdorff space, there exist $O^{\prime}, O^{\prime \prime} \in \tau(X)$ such that $x \in O^{\prime}$, $X \backslash O \subset O^{\prime \prime}$, and $O^{\prime} \cap O^{\prime \prime}=\varnothing$.

Then, for the closed set $B:=X \backslash O^{\prime \prime}$ and the neighborhood $O^{\prime}$, we have the required relations: $x \in O^{\prime} \subset B \subset O$.

Lemma 2. Let $X$ be a compact Hausdorff space. Let $x \in X, O \in \tau(X)$, and a closed set $B \in X$ be such that $x \in O \subset B$. Then there exists a covering $\left(O_{\iota}\right)_{I} \in O_{\mathrm{fc}}(X)$ such that, for some $\bar{\iota} \in I$, we have

$$
B=O_{\bar{\iota}}, \quad x \in X \backslash \bigcup_{\iota \in I \backslash\{\bar{\iota}\}} O_{\iota} .
$$


Proof. Let $x \in X$ and $O, B \in 2^{X}$ satisfy the conditions of the lemma. Using the Hausdorff property of $X$, for any point $y \in X \backslash\{x\}$, construct neighborhoods $O_{x y}, O_{y x} \in \tau(X)$ such that $x \in O_{x y} \subset O, y \in O_{y x}$, and $O_{x y} \cap O_{y x}=\varnothing$.

Using the compactness of $X$, we select a finite subcovering $\left\{O_{x y_{i}}, O_{y_{j} x} \mid i \in I, j \in J\right\}$ from the open covering $\left\{O_{x y}, O_{y x} \mid y \in X \backslash\{x\}\right\}$ and consider the finite family of closed sets $\left\{B, B_{j} \mid j \in J\right\}$, where $B_{j}$ denotes the closure of the set $O_{y_{j} x}$. Since $O_{x y_{i}} \subset O \subset B$, $i \in I$, this family is a covering of $X$. By construction, $\left(\bigcup_{j \in J} O_{y_{j} x}\right) \cap\left(\bigcap_{i \in I} O_{x y_{i}}\right)=\varnothing$. Hence, taking into account that $x \in \bigcap_{i \in I} O_{x y_{i}}$, we obtain $x \in X \backslash \bigcup_{j \in J} B_{j}$. We have verified the last of the required properties of the covering $\left\{B, B_{j} \mid j \in J\right\}$.

3. Let us prove inclusion (18) by contradiction: let $x$ belong to the right-hand side of (18) and $x \notin F(x)$. Since the graph of $F$ is closed, the set $F(x)$ is closed. Therefore, in view of the fact that $X$ is a compact Hausdorff space, there are disjoint neighborhoods $O^{\prime}(x), O^{\prime}(F(x)) \in \tau(X)$ of the point $x$ and of the set $F(x): O^{\prime}(x) \cap O^{\prime}(F(x))=\varnothing$. The closedness of the graph of $F$ and the conditions on $X$ imply that the mapping $F$ is upper semicontinuous with respect to inclusion (see [10, Sect. 43, I, Theorem 4] and [11, Theorem 1.2.32]), i.e., there exists a neighborhood $O^{\prime \prime}(x) \in \tau(X)$ such that $\bigcup_{y \in O^{\prime \prime}(x)} F(y) \subset$ $O^{\prime}(F(x))$.

Let, by Lemma 1, an open neighborhood $O$ and a closed set $B$ such that $x \in O \subset B \subset$ $O^{\prime}(x) \cap O^{\prime \prime}(x)$ be found. Let, by Lemma 2, a covering $\left(O_{\iota}\right)_{I} \in O_{\mathrm{fc}}(X)$ satisfy conditions (25). Then these conditions, the assumption $x \in \bigcap_{\left(O_{\iota}\right)_{I} \in O_{\mathrm{fc}}(X)} \bigcup_{\iota \in I} \hat{F}\left(O_{\iota}\right)$, and property (4) imply that

$$
x \in\left(\bigcup_{\iota \in I} \hat{F}\left(O_{\iota}\right)\right) \backslash\left(\bigcup_{\iota \in I \backslash\{\bar{\iota}\}} O_{\iota}\right) \subset\left(\bigcup_{\iota \in I} \hat{F}\left(O_{\iota}\right)\right) \backslash\left(\bigcup_{\iota \in I \backslash\{\bar{\iota}\}} \hat{F}\left(O_{\iota}\right)\right) \subset \hat{F}\left(O_{\bar{\iota}}\right)=\hat{F}(B) .
$$

On the other hand, it follows from the construction of $B$ that

$$
\hat{F}(B):=B \bigcap\left(\bigcup_{y \in B} F(y)\right) \subset O^{\prime}(x) \bigcap\left(\bigcup_{y \in O^{\prime \prime}(x)} F(y)\right) \subset O^{\prime}(x) \bigcap O^{\prime}(F(x))=\varnothing .
$$

We obtain the contradiction: $x \in \varnothing$. The first part of the theorem is proved.

4. For the proof of the second part of the theorem, first we will establish that the sets

$$
\bigcup_{\iota \in I} \hat{F}\left(O_{\iota}\right), \quad\left(O_{\iota}\right)_{I} \in O_{\mathrm{fc}}(X)
$$

are closed and centered if condition (9) is satisfied. This property along with the compactness of the set $X$ will imply the nonemptiness of the right-hand side of (18).

The closedness of these sets follows from the definitions of the family $O_{\mathrm{fc}}(X)$ and the mapping $\hat{F}$ and from the closedness of the mapping $F$ (see [11, Theorem 1.2.33]): for any closed set $Y \subset X$, the set $\cup_{y \in Y} F(y)$ is closed.

Let us verify that sets $(26)$ are centered. Let $\left(O_{k \iota}\right)_{I_{k}} \in O_{\mathrm{fc}}(X), k \in 1 . . n, n \in \mathbb{N}$. We define $\left(\bar{O}_{\bar{\iota}}\right)_{\bar{I}}:=\left\{O_{1 \iota_{1}} \cap \ldots \cap O_{n \iota_{n}} \mid \iota_{k} \in I_{k}, k \in 1 . . n\right\}$. In other words, $\left(\bar{O}_{\bar{\iota}}\right)_{\bar{I}}$ is the greatest lower bound of the finite set of covering $\left(O_{k \iota}\right)_{I_{k}}$ with respect to the inscribing relation. By 
construction, $\left(\bar{O}_{\bar{\iota}}\right)_{\bar{I}} \in O_{\mathrm{fc}}(X)$ and is inscribed into all the families $\left(O_{k \iota}\right)_{I_{k}}:\left(\bar{O}_{\bar{\iota}}\right)_{\bar{I}} \sqsubseteq\left(O_{k \iota}\right)_{I_{k}}$, $k \in 1 . . n$. Therefore (see (6)), for any $k \in 1 . . n$, the inclusion $\bigcup_{\bar{\iota} \in \bar{I}} \hat{F}\left(\bar{O}_{\bar{\iota}}\right) \subset \bigcup_{\iota \in I_{k}} \hat{F}\left(O_{k \iota}\right)$ holds. Hence, in view of the condition $\bigcup_{\bar{\iota} \in \bar{I}} \hat{F}\left(\bar{O}_{\iota}\right) \neq \varnothing$, we obtain $\varnothing \neq \bigcup_{\bar{\iota} \in \bar{I}} \hat{F}\left(\bar{O}_{\bar{\iota}}\right) \subset$ $\bigcap_{k \in 1 . . n} \bigcup_{\iota \in I_{k}} \hat{F}\left(O_{k \iota}\right)$. Thus, subsets $(26)$ of the compact space $X$ are closed and centered. Consequently, $\boldsymbol{F i x}(F)=\bigcap_{\left(O_{\iota}\right)_{I} \in O_{\mathrm{fc}}(X)} \bigcup_{\iota \in I} \hat{F}\left(O_{\iota}\right) \neq \varnothing$.

Conversely, let $\bar{x} \in \mathbf{F i x}(F)$. Then, for any covering $\left(O_{\iota}\right)_{I} \in O(X)$, there exists a set $O_{\bar{\iota}} \in\left(O_{\iota}\right)_{I}$ such that $\bar{x} \in O_{\bar{\iota}}$. Hence, by definition, $\bar{x} \in \hat{F}\left(O_{\bar{\iota}}\right)$. Therefore, $\hat{F}\left(O_{\bar{\iota}}\right) \neq \varnothing$.

For the justification of Corollary 1, we note that the condition of closedness of the mapping graph is equivalent to the condition of function continuity.

\subsection{Proof of Theorem 3 and Corollary 2}

In this subsection, for arbitrary $\delta>0, x \in X$, and $Y \in 2^{X}$, we define

$$
O_{\delta}(x):=\{y \in X \mid \rho(x, y) \leqslant \delta\}, \quad O_{\delta}(Y):=\{y \in X \mid \mathbf{d}(y, Y) \leqslant \delta\} .
$$

1. By virtue of Theorem 1 and the inclusions $\left(O_{k \iota}\right)_{I_{k}} \subset O(X), k \in \mathbb{N}$, for the proof of the first part, it suffices to establish that $\operatorname{Fix}(F) \supset \bigcap_{k \in \mathbb{N}} \bigcup_{\iota \in I_{k}} \hat{F}\left(O_{k \iota}\right)$.

Assume that $\bar{x} \in \bigcap_{k \in \mathbb{N}} \bigcup_{\iota \in I_{k}} \hat{F}\left(O_{k \iota}\right)$. Then there is a sequence

$$
\bar{O}_{k}:=O_{k \iota_{k}}, \quad \bar{x} \in \hat{F}\left(\bar{O}_{k}\right) \subset \bar{O}_{k}, \quad \operatorname{diam}\left(\bar{O}_{k}\right) \leqslant \delta_{k}, \quad k \in \mathbb{N} .
$$

Let us show that $\bar{x} \in \mathbf{F i x}(F)$. Suppose the contrary: $\bar{x} \notin F(\bar{x})$. Define $\bar{\varepsilon}:=\mathbf{d}(\bar{x}, F(\bar{x})) / 3$. Since the graph of $F$ is closed, the set $F(\bar{x})$ is closed. Therefore, $\bar{\varepsilon}>0$. Choose $\bar{\delta} \in(0, \bar{\varepsilon}]$ such that the relations $F(x) \subset O_{\bar{\varepsilon}}(F(\bar{x}))$ hold for all $x \in O_{\bar{\delta}}(\bar{x})$. We can make this, since the closedness of the graph of $F$ yields the upper semicontinuity of the mapping $F$ with respect to inclusion (see [10, Sect. 43, I, Theorem 4] and [11, Theorem 1.2.32]). At the same time, due to the choice of the values $\bar{\delta}$ and $\bar{\varepsilon}$, we have $O_{\bar{\delta}}(\bar{x}) \bigcap O_{\bar{\varepsilon}}(F(\bar{x}))=\varnothing$. From these constructions, we obtain

$$
\hat{F}\left(O_{\bar{\delta}}(\bar{x})\right):=O_{\bar{\delta}}(\bar{x}) \bigcap\left(\bigcup_{x \in O_{\bar{\delta}}(\bar{x})} F(x)\right) \subset O_{\bar{\delta}}(\bar{x}) \bigcap O_{\bar{\varepsilon}}(F(\bar{x}))=\varnothing .
$$

In view of (10), choose $\bar{k} \in \mathbb{N}$ such that the inclusion $\bar{O}_{\bar{k}} \subset O_{\bar{\delta}}(\bar{x})$ holds. From these inclusions and relations (28) and (2), we obtain $\hat{F}\left(\bar{O}_{\bar{k}}\right) \subset \hat{F}\left(O_{\bar{\delta}}(\bar{x})\right)=\varnothing$, which contradicts (27) for $k=\bar{k}$. The first part of the theorem is proved.

2. Let us consider the second part of the assertion. The necessity of condition (12) follows immediately. Now, we verify the sufficiency of this condition. Using the compactness of $X$, construct a sequence $\left(O_{k \iota}\right)_{I_{k}}, k \in \mathbb{N}$, of inscribed open coverings of the form:

$$
\left(O_{k+1 \iota}\right)_{I_{k+1}} \sqsubseteq\left(O_{k \iota}\right)_{I_{k}}, \quad\left(O_{k \iota}\right)_{I_{k}} \in O_{\mathrm{fo}}^{1 / k}(X), \quad k \in \mathbb{N} .
$$

Then the sequence of closed finite coverings $\left(\bar{O}_{k \iota}\right)_{I_{k}}, k \in \mathbb{N}$, where $\bar{O}_{k \iota}$ is the closure of the set $O_{k \iota}$ in $X$, satisfies conditions (10):

$$
\left(\bar{O}_{k+1 \iota}\right)_{I_{k+1}} \sqsubseteq\left(\bar{O}_{k \iota}\right)_{I_{k}}, \quad\left(\bar{O}_{k \iota}\right)_{I_{k}} \in O_{\mathrm{fc}}^{1 / k}(X), \quad k \in \mathbb{N} .
$$


In this case, by (6), the sequence of the closed sets $\bigcup_{\iota \in I_{k}} \hat{F}\left(\bar{O}_{k \iota}\right)$ is monotonically decreasing by inclusion. Using condition (12), we verify the nonemptiness of the terms of this sequence and, hence, the nonemptiness of their intersection (by a part of the first theorem, this is the set of fixed points of $F$ ).

For any $k \in \mathbb{N}$, take $\delta_{k}$ equal to the half of the Lebesgue number of the covering $\left(O_{k \iota}\right)_{I_{k}}$. Then condition (12) implies the existence of a pair $x_{k}, y_{k} \in X$ such that $\rho\left(x_{k}, y_{k}\right) \leqslant \delta_{k}$ and $y_{k} \in F\left(x_{k}\right)$. Moreover, due to the choice of $\delta_{k}$, there exists an element $O_{k \bar{\iota}}$ of the covering $\left(O_{k \iota}\right)_{I_{k}}$ such that $x_{k}, y_{k} \in O_{k \bar{l}} \subset \bar{O}_{k \bar{\imath}}$. From the last two relations, we obtain $\hat{F}\left(\bar{O}_{k \bar{l}}\right) \neq \varnothing$. Consequently, $\bigcup_{\iota \in I_{k}} \hat{F}\left(\bar{O}_{k \iota}\right) \neq \varnothing$.

For the justification of Corollary 2, note that the condition of closedness of the mapping graph is equivalent to the condition of function continuity.

\subsection{Proof of Theorem 4}

Let us prove the following auxiliary assertion.

Lemma 3. The set of fixed points of mapping $F_{\varphi}$, i.e., the set of pairs $\left(u_{*}, v_{*}\right) \in \mathbf{U} \times \mathbf{V}$ satisfying the condition

$$
\left(u_{*}, v_{*}\right) \in F_{\varphi}\left(u_{*}, v_{*}\right),
$$

coincides with the set of saddle points of the function $\varphi(\cdot)$, i.e., with the set of pairs $\left(u_{*}, v_{*}\right) \in \mathbf{U} \times \mathbf{V}$ satisfying the inequalities

$$
\varphi\left(u_{*}, v\right) \leqslant \varphi\left(u, v_{*}\right) \quad \forall(u, v) \in \mathbf{U} \times \mathbf{V} .
$$

Proof. Assume that inclusion (29) holds. It means that the following inequalities are valid:

$$
\begin{array}{ll}
\varphi\left(u_{*}, v_{*}\right) \leqslant \inf _{u^{\prime} \in \mathbf{U}} \varphi\left(u^{\prime}, v_{*}\right) \leqslant \varphi\left(u, v_{*}\right) & \forall u \in \mathbf{U}, \\
\varphi\left(u_{*}, v\right) \leqslant \sup _{v^{\prime} \in \mathbf{V}} \varphi\left(u_{*}, v^{\prime}\right) \leqslant \varphi\left(u_{*}, v_{*}\right) & \forall v \in \mathbf{V} .
\end{array}
$$

Since the left-hand side of the inequality in (31) equals to the right-hand side of the inequality in (32), we have relations (30) which mean that the pair $\left(u_{*}, v_{*}\right)$ is a saddle point. To complete the proof, note that the above arguments are invertible.

The properties of the function $\varphi(\cdot)$ and of the spaces $\mathbf{U}$ and $\mathbf{V}$ required in the condition of Theorem 4 imply that the graph of the mapping $F_{\varphi}: \mathbf{U} \times \mathbf{V} \mapsto 2^{\mathbf{U} \times \mathbf{V}}$ is closed and $\mathbf{U} \times \mathbf{V}$ is a compact Hausdorff space with the topology of the product of the topological spaces $\mathbf{U}$ and $\mathbf{V}$. Thus, conditions of Theorem 2 are satisfied for the mapping $F_{\varphi}$.

Let the mapping $\hat{F}_{\varphi}$ satisfy condition (9). Then it follows from Theorem 2 and Lemma 3 that the set of saddle points for the function $\varphi$ (30) is nonempty. Hence, equality (14) holds.

Conversely, let equality (14) hold. Then, by virtue of the conditions on the function $\varphi(\cdot)$ and on the spaces $\mathbf{U}$ and $\mathbf{V}$, the set $\operatorname{argmin}_{u \in \mathbf{U}} \max _{v \in \mathbf{V}} \varphi(u, v) \times \operatorname{argmax}_{v \in \mathbf{V}} \min _{u \in \mathbf{U}} \varphi(u, v)$ is nonempty and coincides with the set of saddle points of the mapping $F_{\varphi}$. In this case, Lemma 3 and Theorem 2 imply that condition (9) holds. 


\subsection{Scheme of the Proof of Theorem 5}

The lemma given below follows from the definition of mapping $F$.

Lemma 4. $N(J)=\mathbf{F i x}\left(F_{(X, J)}\right)$.

The properties of functions $J_{i}$ and spaces $X_{i}, i \in 1 . . n$, required in the condition of Theorem 5 imply that the graph of mapping $F_{(X, J)}: X \mapsto 2^{X}$ is closed and $X$ is a compact Hausdorff space. Thus, conditions of Theorem 2 are satisfied for the space $X$ and the mapping $F_{(X, J)}$.

After this remark, it is easy to see that the first part of Theorem 5 follows from Lemma 4 and Theorem 2. The equivalence of conditions (16) and (9) follows from the definition of function $\hat{F}_{(X, J)}$ by mapping $F_{(X, J)}$.

\section{References / Литература}

1. Kakutani S. A Generalization of Brouwer's Fixed Point Theorem. Duke Mathematical Journal, 1941, vol. 8, pp. 457-459. DOI: 10.1215/S0012-7094-41-00838-4

2. Park Sehie. Recent Results in Analytical Fixed Point Theory. Nonlinear Analysis, 2005, vol. 63, pp. 977-986. DOI: 10.1016/j.na.2005.02.026

3. Tarski A.A Lattice-Theoretical Fixpoint Theorem and Its Applications. Pacific Journal of Mathematics, 1955, vol. 5, no. 2, pp. 285-309. DOI: 10.2140/pjm.1955.5.285

4. Kantorovitch L. The Method of Successive Approximation for Functional Equations. Acta Mathematica, 1939, December, vol. 71, no. 1, pp. 63-97. DOI: 10.1007/BF02547750

5. Barendregt H. P. Lambda Calculus. Its Syntax and Semantics. North-Holland Publishing Company, 1981.

6. Li Jinlu Several Extensions of the Abian-Brown Fixed Point Theorem and Their Applications to Extended and Generalized Nash Equilibria on Chain-Complete Posets. Journal of Mathematical Analysis and Applications, 2014, vol. 409, no. 2, pp. 1084-1092. DOI: $10.1016 /$ j.jmaa.2013.07.070

7. Fan K. Minimax theorems. Proceedings of the National Academy of Sciences U.S.A., 1953, vol. 39 , pp. $42-47$. DOI: $10.1073 /$ pnas.39.1.42

8. Nadler S.B.Jr. Multi-Valued Contraction Mappings. Pacific J. Math, 1969, vol. 30, pp. $475-488$. DOI: $10.2140 /$ pjm.1969.30.475

9. Arutyunov A.V. Covering Mappings in Metric Spaces and Fixed Points. Doklady Mathematics, 2007, vol. 76, no. 2, pp. 665-668. DOI:10.1134/S1064562407050079

10. Kuratowski K. Topology. Volume II. Academic Press, New York, 1968.

11. Borisovich Yu.G., Gel'man B.D., Myshkis A.D. etc. Vvedeniye v teoriyu mnogoznachnykh otobrazheniy $i$ differentsial'nykh vklyucheniy [Introduction to the Theory of Multi-Valued Mappings and Differential Inclusions]. Moscow, Librokom, 2011. 224 р. [Введение в теорию многозначных отображений и дифференциальных включений / Ю.Г. Борисович, Б.Д. Гельман, А.Д. Мышкис и др. - М.: Либроком, 2011. - 224 с.]

Received June 30, 2015 


\title{
К ТЕОРИИ НЕПОДВИЖНЫХ ТОЧЕК И ЕЕ ПРИЛОЖЕНИЙ К МОДЕЛЯМ РАВНОВЕСИЙ
}

\author{
Д.А. Серков
}

\begin{abstract}
Для заданных множества и (вообще говоря, многозначного) отображения этого множества в себя рассматривается вопрос о существовании неподвижных точек такого отображения, то есть точек, содержащихся в своем образе. Относительно заданных множества и отображения предполагается, что множество не пусто, а отображение определено на всем множестве. В этих условиях дается описание (переопределение) множества неподвижных точек в теоретико-множественных терминах. Это общее представление конкретизируется для случаев, когда множество наделено той или иной топологической структурой, а отображение имеет дополнительные свойства с ней связанные. В частности, предложены необходимые и достаточные условия существования неподвижных точек для случая отображений с замкнутым графиком как в хаусдорфовых топологических пространствах, так и в метрических пространствах. Приведен пример, иллюстрирующий возможности и преимущества предлагаемого подхода. Также даны непосредственные приложения этих результатов к поиску равновесных состояний в игровых задачах: описаны множества седловых точек (аналог теоремы Фана) в задаче о минимаксе и точек равновесия по Нэшу в игре со многими участниками для случаев, когда множества стратегий игроков являются хаусдорфовыми или метризуемыми топологическими пространствами.
\end{abstract}

Ключевые слова: многозначное отображение; неподвижная точка; седловая точка; равновесие по Нэшу.

Дмитрий Александрович Серков, доктор физико-математических наук, ведущий научный сотрудник, Институт математики и механики им. Н.Н. Красовского УрО РАН; профессор, кафедра «Вычислительные методы и уравнения математической физики», Институт радиоэлектроники и информационных технологий, Уральский федеральный университет им. Б.Н. Ельцина, (г. Екатеринбург, Россия), serkov@imm.uran.ru.

Поступила в редакцию 30 июня 2015 г. 